\title{
Absorption Spectra of Methane in the Near Infrared
}

\author{
By Richard C. Nelson, ${ }^{1}$ Earle K. Plyler, and William S. Benedict
}

\begin{abstract}
A grating spectrometer with a $\mathrm{PbS}$ cell for detector has been used for the measurement of the infrared absorption bands of methane in the region of $1.66 \mu$. Many lines of the $P$, $Q$, and $R$ branches of the $1.66-\mu$ band have been observed.

These are well resolved and are of the correct structure and spacing for the $\mathrm{F}_{2}$ component of $2 \nu_{3}$. The lines for values of $J$ up to 10 are sharp, showing no indication of splitting due to interaction with neighboring states or to centrifugal distortion. The rotational constants obtained for this band are $B^{\prime}=5.178, \zeta_{3}=0.0346$. The latter value is lower than in the $\nu_{3}$ fundamental. Other bands observed are more complex and irregular, presumably due to mutual interaction.
\end{abstract}

In the infrared spectrum of methane there are two strong bands, the fundamental frequencies, $\nu_{3}$ at $3020.3 \mathrm{~cm}^{-1}$, and $\nu_{4}$ at $1306.2 \mathrm{~cm}^{-1}$. Because of the symmetry of the molecule, the other two fundamentals $\nu_{1}$ at $2914.2 \mathrm{~cm}^{-1}$ and $\nu_{2}$ at 1526 $\mathrm{cm}^{-1}$, are not active in the infrared, although these frequencies may appear in combination bands with the other frequencies and their overtones, to give bands in the near infrared. Many previous studies, both experimental and theoretical, have been made of the methane molecule. A good discussion of the general problem, including references to the literature, may be found in Herzberg's book [1]. ${ }^{2}$ One of the unsettled problems concerns the influence of the Coriolis forces in the fine structure of the overtone and combination bands. Accordingly it was thought that further experimental data with higher resolution would be of value. The present paper reports such data in the regions from 1.5 to $1.7 \mu$, and from 1.15 to $1.19 \mu$, with particular emphasis on one band of relatively simple structure, the $F_{2}$ component of $2 \nu_{3}$, at about $6,004 \mathrm{~cm}^{-1}$. Quite recent studies on the atmospheric absorption have revealed the presence of methane bands [2]. McMath, Mohler, and Goldberg [2] have also studied the $2 \nu_{3}$ band under high resolution.

In our studies, high resolution was obtained by the use of a specially designed grating instrument, which employs a PbS photo-conducting cell con-

\footnotetext{
1 Research Associate, Northwestern University.

${ }^{2}$ Figures in brackets indicate the literature references at the end of this paper.
}

structed by Cashman [3] as the detecting unit. One of the authors [4] has previously given a description of the instrument and only a brief outline of the arrangement will be repeated here.

Figure 1 shows the plan of the optical parts of the spectrometer. The system is designed according to the arrangement described by Pfund [5]. The paraboloidal mirrors have a focal length of $2 \mathrm{~m}$ and are $12 \mathrm{in}$. in diameter. The grating has 15,000 lines per inch and the ruled surface is 3 by 5 in., giving a theoretical resolution in the first order of 75,000. An ellipsoidal mirror, with a conjugate focal ratio of $1: 3$, was used to focus the energy from the exit slit onto the $\mathrm{PbS}$ cell. With this magnification, spectrometer slits $18 \mathrm{~mm}$ in length could be used with the PbS cell, which possessed a sensitive surface $7 \mathrm{~mm}$ long. A tungsten lamp was used as the source, and a quartz lens focused the filament on the entrance slit of the spectrometer. The absorption cell, which was $60 \mathrm{~cm}$ in length, was placed in front of the entrance slit. A chopper, which interrupts the radiant energy approximately 1,000 times a second, was placed immediately in front of the entrance slit.

For any selected region the grating was rotated continuously. The driving mechanism has five scanning speeds, and the Speedomax recorder has six speeds for the chart, a selection which made it possible to have dispersions of 50 to $0.02 \mathrm{~A} / \mathrm{mm}$ on the recording chart.

A special narrow-band-pass amplifier was used with the $\mathrm{PbS}$ cell. The over-all voltage gain was 


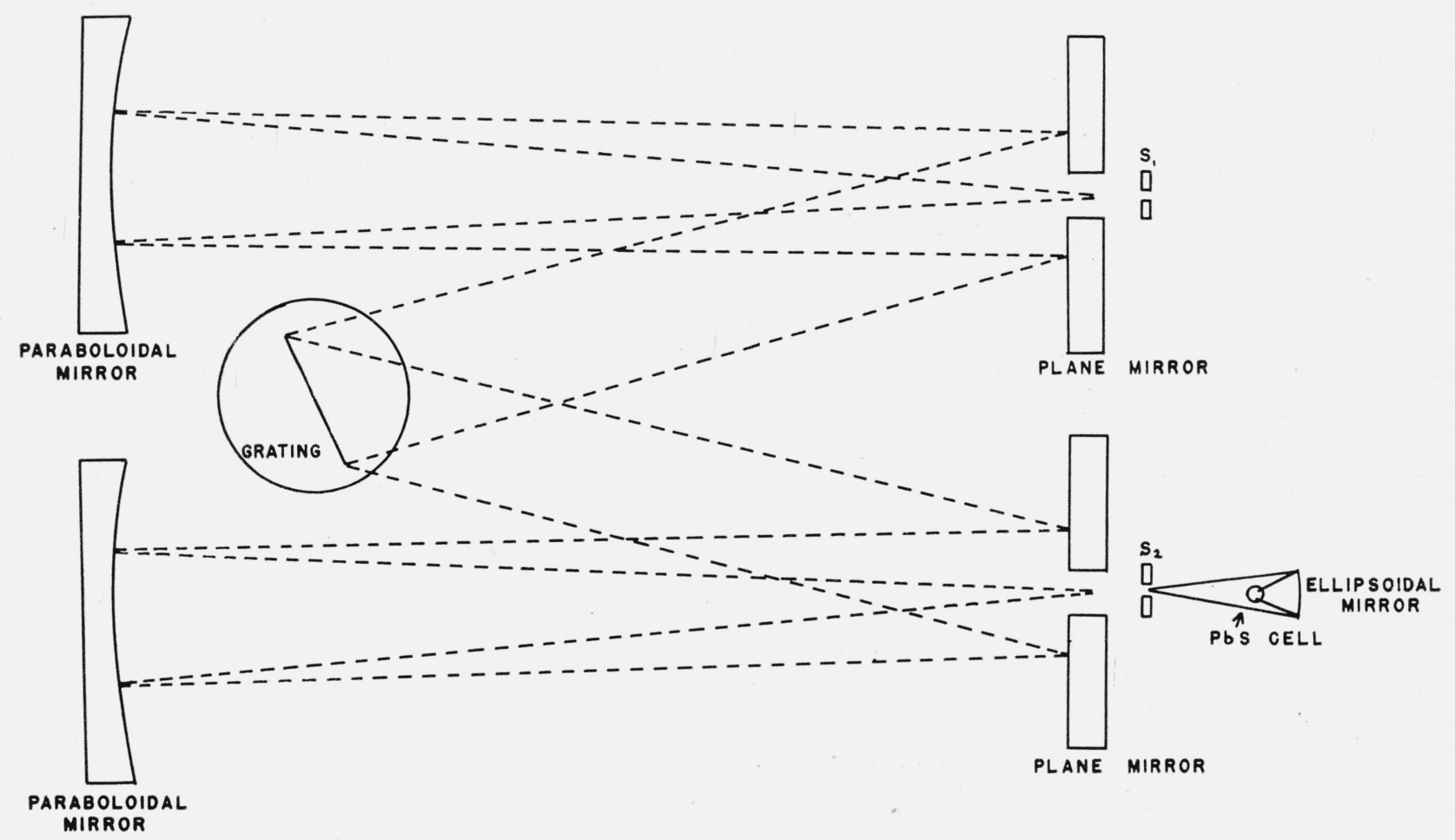

Figure 1. Arrangement of the optical components of the spectrometer.

approximately $1.8 \times 10^{8}$. The details of the amplifier have been given in a previous publication [4].

In the region around $1.66 \mu$, runs were made at two dispersion scales on the recorder chart. Measured wavelengths are largely based on two runs made with slits $0.7 \mathrm{~cm}^{-1}$ spectral width, the dispersion on the chart being about $15 \mathrm{~cm}^{-1} /$ in A commutator in the gear train driving the grating actuated a pen that placed marks on the paper corresponding to equal increments of motion of the nut upon the tangent screw, which moves the grating.

Wavelengths were measured with reference to lines of the $\mathrm{Hg}$ spectrum. A record of the spectrum in all orders over the desired region was made. Then using the lines 15295.2, 5460.74 (3d order), and $4358.35 \mathrm{~A}$ (4th order), a quadratic expression was obtained for wavelength in terms of measurements on the scale impressed on the record. An equation of the form $\lambda_{1}=\lambda_{0}+d a+d^{2} b$ is obtained, where $\lambda_{1}$ is the wavelength corresponding to any number $d$, representing the difference between respective scale readings for $\lambda_{1}$ and $\lambda_{0}$. The $\lambda_{0}, a$, and $b$ are constants that are determined from the scale readings of the three lines used for calibration. During continuous operation of the instrument, the line $15295.2 \mathrm{~A}$ was recorded, followed by the absorption spectrum of $\mathrm{CH}_{4}$, and the record was completed by recording $4358.35 \times 4$. The measured separations of the initial and final $\mathrm{Hg}$ lines were alike, and the previously found expression was used for computing wavelengths of absorption lines.

Another run was made with slits $0.3 \mathrm{~cm}^{-1}$ spectral width, and dispersion on the chart of about $5 \mathrm{~cm}^{-1} / \mathrm{in}$. to bring out fine detail.

Measurements were made both on natural gas $\left(80 \% \mathrm{CH}_{4}\right)$ and Matheson $\mathrm{CH}_{4}, 99$ percent pure, in a cell $60 \mathrm{~cm}$ long. The light source in all cases was a tungsten filament.

Figure 2 shows the results for the $1.66-\mu$ region. The strong rotational lines of the $P$ and $R$ branches of the $2 \nu_{3}$ band of methane can readily be identified. About 19 lines are observed for the $R$ branch with the $60-\mathrm{cm}$ cell at atmospheric pressure. In addition there are present a number of less intense lines, which appear between these lines. The less intense lines repeat well on successive runs. Beyond the 10th line of the $P$ branch, the structure becomes complex. This structure may be part of another band. There is a narrow region of the spectrum without any lines, followed by another set of com- 


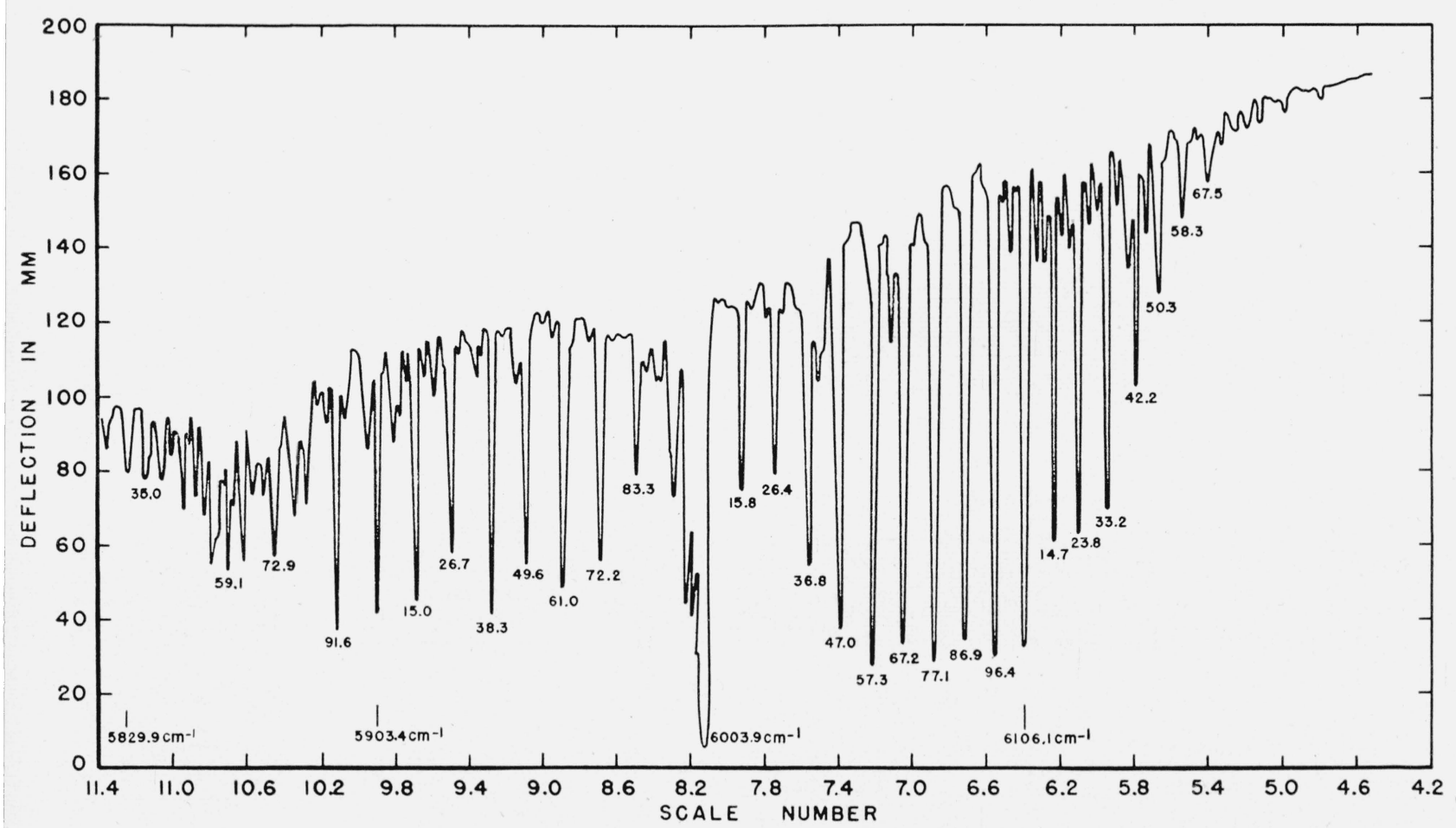

Figure 2. Absorption spectrum of the 1.66- $\mu$ band $\mathrm{CH}_{4}$.

The cell was $60 \mathrm{~cm}$ in length and the gas at atmospheric pressuré.

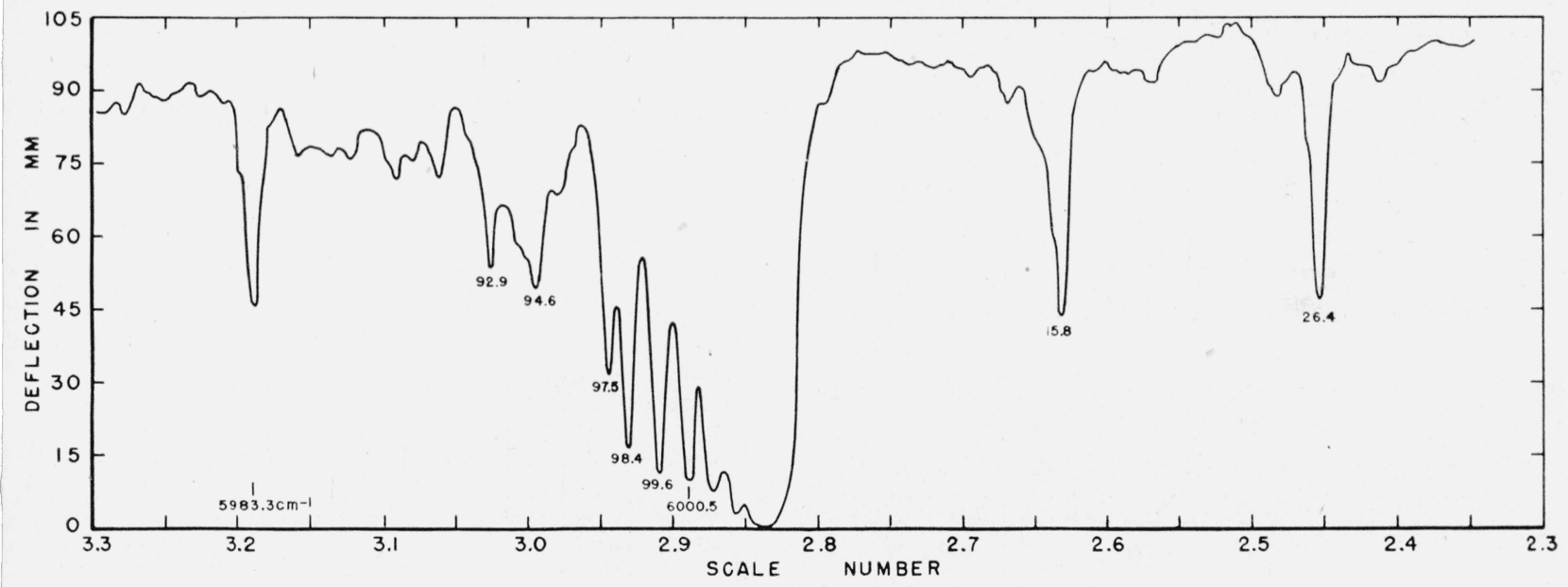

Figure 3. Center of the 1.66- $\mu$ band, showing fine structure states of the zero branch.

plex lines. These lines may be part of a combination band, but no term assignment fits very well. When the absorption of the gas was measured with a spectral slit of $0.3 \mathrm{~cm}^{-1}$, the zero branch showed more structure. Figure 3 shows the central part of the band. The gas was at atmospheric pressure for these observations. When a smaller pressure of gas was used, better separation of the stronger components of the $Q$ branch was obtained, but the less intense components were not present.
The intensities given are measured to within 2 percent. The stronger lines may actually be more intense than the measurements indicate. That is, the finite slit widths may make it impossible to find the maximum absorption at the center of the lines. All faint lines that measure less than 3 percent are arbitrarily marked 2 percent in intensity. The intensity figures in the table denote the percentage absorption of the minimum point of the lines. 
All the observed lines of appreciable intensity for the region are listed in table 1 . Wave numbers of some of the lines are marked for identification. A sufficient number of lines are numbered on the figure so that the tables can be related to the experimental results. The band for the $1.10-$ to $1.13-\mu$ region, shown in figure 4 , does not have the regular structure as observed in the $2 \nu_{3}$ band. The wavelengths and intensities of all the lines are given in table 2 .

The $2 \nu_{3}$ band gives sufficient data for calculating some of the constants of methane. The only lines in the $\mathrm{CH}_{4}$ absorption that can be given a definite interpretation are listed in table 3 . These are the strongest lines in the region, and form a band consisting of $P, Q$, and $R$ branches of simple structure, the band bearing a marked resemblance to the $\nu_{3}$ fundamental. The vibrational transition involved, $2 \nu_{3}$, is the first overtone of that fundamental. The theory of the structure of this band has been given by Johnston and Dennison, [6] and by Shaffer, Nielsen, and Thomas [7]. The firstnamed authors have pointed out that the upper level is six-fold degenerate in the zeroth-order approximation, but that the first-order Coriolis interaction due to the internal angular momentum of the vibration removes the degeneracy, giving six different energies for each value of the rotational quantum number $J$. At $J=0$, these may be grouped into three sub-bands of different symmetry. Transitions are permitted from the ground state to only one of these sub-bands, the triply-degenerate $F_{2}$; and for each branch, $\Delta J=$ $-1,0$, or +1 , transitions are permitted to only one of the three components, thus giving the band of simple structure, as observed.

The second-named authors have considered the effect of second-order interactions, which in principle may further remove the degeneracy of the energy levels, splitting each $J$ level into a number of components of different $K$, and which may permit transitions from the ground state to the $E$ subband. (These authors gave selection rules and intensities for the band which differ from those of Johnston and Dennison [6], and which appear to be in error.) The second-order interactions are of two types; first, those due to centrifugal stretching and distortion of the molecule; and second, those due to resonance interactions between the vibrational level and other neighboring levels of the same total (vibrational plus
TABLE 1. Wavelength, wave number, and intensity of the observed lines in the $1.66-\mu$ region

\begin{tabular}{|c|c|c|c|c|c|}
\hline $\begin{array}{l}\text { Wave- } \\
\text { length }\end{array}$ & $\begin{array}{l}\text { Wave } \\
\text { number }\end{array}$ & $\begin{array}{c}\text { Absorp- } \\
\text { tion }\end{array}$ & $\begin{array}{l}\text { Wave- } \\
\text { length }\end{array}$ & $\begin{array}{l}\text { Wave } \\
\text { number }\end{array}$ & $\begin{array}{c}\text { Absorp } \\
\text { tion }\end{array}$ \\
\hline A & $\mathrm{cm}^{-1}$ & Percent & A & $\mathrm{cm}^{-1}$ & Percent \\
\hline 16112.7 & 6204.6 & 3 & 16859.9 & 5929.6 & 5 \\
\hline 16143.6 & 6192.7 & 2 & 16868.0 & 5926.7 & 50 \\
\hline 16148.5 & 6190.8 & 2 & 16884.7 & 5920.9 & 10 \\
\hline 16165.9 & 6184.1 & 2 & 16894.6 & 5917.4 & 5 \\
\hline 16175.6 & 6180.4 & 2 & 16901.4 & 5915.0 & 50 \\
\hline 16185.3 & 6176.7 & 2 & 16918.9 & 5918.9 & 20 \\
\hline 16200.3 & 6171.0 & 2 & 16934.8 & 5903.4 & 50 \\
\hline 16209.6 & 6167.5 & 5 & 16943.2 & 5900.4 & 20 \\
\hline 16233.7 & 6158.3 & 10 & 16968.5 & 5891.6 & 55 \\
\hline 16254.8 & 6150.3 & 20 & 16977.2 & 5888.6 & 10 \\
\hline 16260.2 & 6148.2 & 5 & 16995.3 & 5882.4 & 25 \\
\hline 16267.2 & 6145.6 & 30 & 17003.6 & 5879.5 & 25 \\
\hline 16276. 2 & 6142.2 & 30 & 17022.7 & 5872.9 & 30 \\
\hline 16281.4 & 6140.3 & 15 & 17030.6 & 5870.2 & 20 \\
\hline 16288.2 & 6137.1 & 10 & 17040.1 & 5866.9 & 15 \\
\hline 16300.2 & 6133.2 & 50 & 17048.4 & 5864.1 & 30 \\
\hline 16309.6 & 6129.7 & 5 & 17062.9 & 5859.1 & 30 \\
\hline 16315.3 & 6127.3 & 5 & 17076.4 & 5854.4 & 30 \\
\hline 16325.1 & 6123.8 & 50 & 17081.8 & 5857.6 & 20 \\
\hline 16331.4 & 6121.5 & 10 & 17090.1 & 5849.7 & 15 \\
\hline 16338.6 & 6118.8 & 10 & 17100.5 & 5846.2 & 20 \\
\hline 16349.4 & 6114.7 & 55 & 17110.6 & 5842.7 & 10 \\
\hline 16356.0 & 6112.3 & 10 & 17117. 2 & 5840.5 & 15 \\
\hline 16362.2 & 6110.0 & 10 & 17133.3 & 5835.0 & 15 \\
\hline 16372.6 & 6106.1 & 70 & 17148.3 & 5829.9 & 15 \\
\hline & & & 17164.7 & 5824.3 & 20 \\
\hline 16386.6 & 6100.9 & 10 & 17200.9 & 5812.0 & 15 \\
\hline 16398.7 & 6096.4 & 70 & 17209.4 & 5809.2 & 10 \\
\hline 16424.2 & 6086.9 & 70 & 17217. 2 & 5806.5 & 10 \\
\hline 16450.6 & 6077.1 & 75 & 17222.6 & 5804.7 & 20 \\
\hline 16477.4 & 6067.2 & 65 & 17232.6 & 5801.3 & 10 \\
\hline 16488.6 & 6063.1 & 15 & 17244.2 & 5797.5 & 10 \\
\hline 16504.5 & 6057.3 & 70 & 17256.3 & 5793.4 & 15 \\
\hline 16532.5 & 6047.0 & 65 & 17265.3 & 5790.4 & 25 \\
\hline 16553.1 & 6039.5 & 15 & 17279.3 & 5785.7 & 20 \\
\hline \multirow[t]{2}{*}{16560.5} & \multirow[t]{2}{*}{6036.8} & \multirow[t]{2}{*}{50} & 17304.1 & 5777.4 & 25 \\
\hline & & & 17315.1 & 5773.7 & 15 \\
\hline 16589.0 & 6026.4 & 30 & 17345.5 & 5763.6 & 15 \\
\hline 16618.4 & 6015.8 & 30 & 17378.5 & 5752.6 & 15 \\
\hline 16651.1 & 6003.9 & 85 & 17395.5 & $5747 . \mathrm{C}$ & 10 \\
\hline 16659.8 & 60001.8 & 65 & \multirow{2}{*}{$\begin{array}{l}17415.1 \\
17456.1\end{array}$} & \multirow{2}{*}{$\begin{array}{l}5740.5 \\
5727.1\end{array}$} & \multirow{2}{*}{$\begin{array}{l}10 \\
15\end{array}$} \\
\hline 16663.2 & 5999.6 & 55 & & & \\
\hline \multirow{2}{*}{$\begin{array}{l}16666.5 \\
16677.1\end{array}$} & $\begin{array}{l}5998.4 \\
5994.6\end{array}$ & \multirow{2}{*}{$\begin{array}{l}50 \\
25\end{array}$} & \multirow{2}{*}{\multicolumn{3}{|c|}{ Q BRANCH * }} \\
\hline & 5994.6 & & & & \\
\hline 16681.8 & 5992.9 & 20 & & & \\
\hline 16688.3 & 5990.6 & 5 & & 6004.2 & 100 \\
\hline 16692.9 & 5988.9 & 5 & $\begin{array}{l}16650.4 \\
16651.1\end{array}$ & $\begin{array}{l}6004.2 \\
6003.9\end{array}$ & 100 \\
\hline & & & 16652.7 & 6003.4 & 100 \\
\hline 16708.5 & 5983.3 & 25 & 16654.7 & 6002.7 & 100 \\
\hline 16725. 2 & 5977.4 & 5 & 16656.9 & 6001.9 & 90 \\
\hline 16739.5 & 5972.2 & 45 & & & \\
\hline 16771.1 & 5961.0 & 50 & 16659.8 & 6000.8 & 90 \\
\hline 16781.3 & 5957.4 & 70 & 16663.2 & 5999.6 & 90 \\
\hline & & & 16666.5 & 5998.4 & 80 \\
\hline 16803.1 & 5949.6 & 45 & 16669.0 & 5997.5 & 65 \\
\hline 16811.3 & 5946.7 & 15 & 16674.3 & 5995.6 & 25 \\
\hline 16826.0 & 5941.5 & 5 & & & \\
\hline 16835.2 & 5938.3 & 50 & 16677.1 & 5994.6 & 45 \\
\hline 16848.5 & 5933.6 & 5 & 16681.8 & 5992. 9 & 40 \\
\hline
\end{tabular}

s These data refer to fig. 3 . 


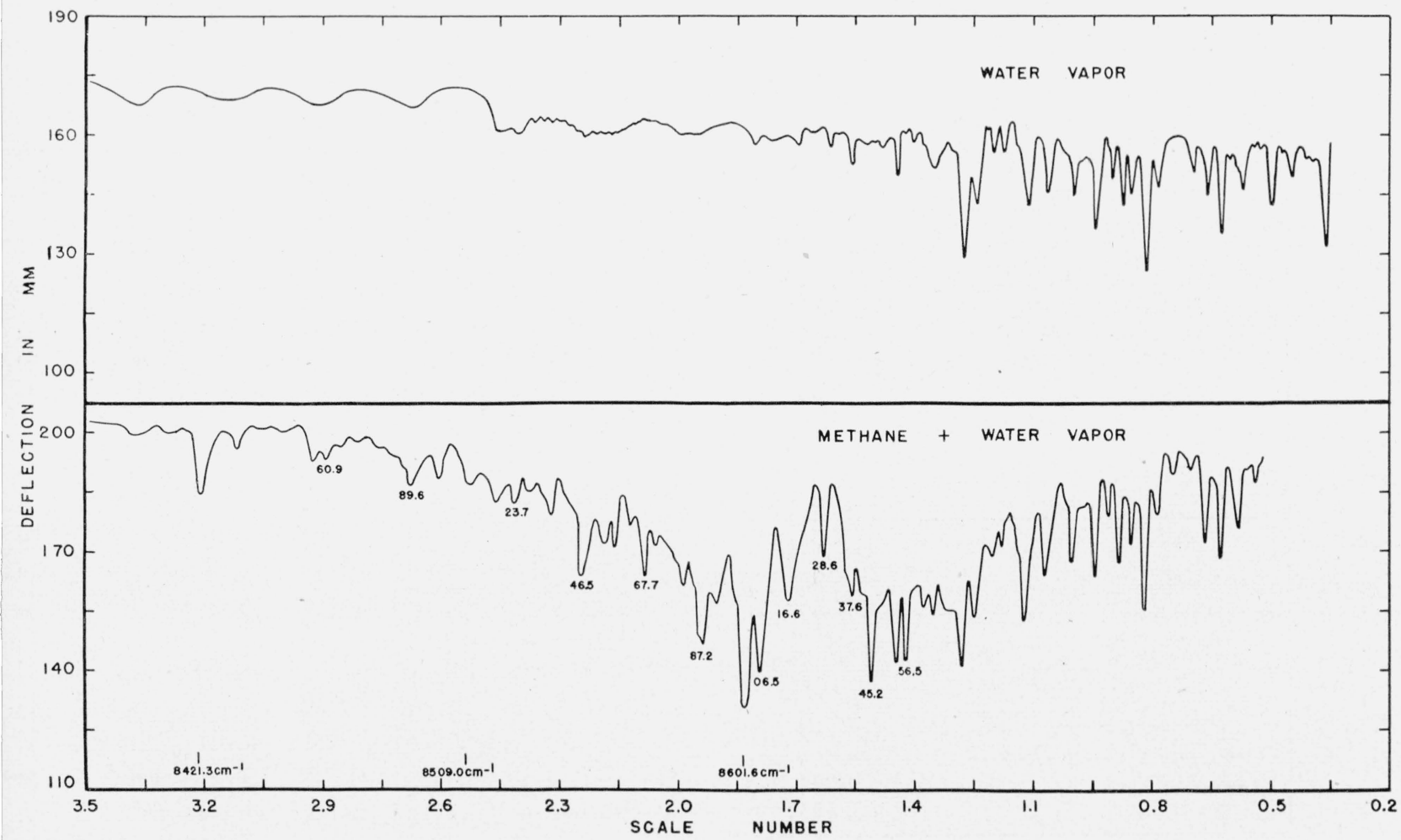

FIGURE 4. Upper curve shows the absorption of water vapor in the atmosphere; lower curve is the absorption of methane interposed on the atmospheric absorption.

TABLE 2. Wavelength, wave number, and intensity of observed lines in the $1.16-\mu$ region

\begin{tabular}{|c|c|c|c|c|c|}
\hline $\begin{array}{c}\text { Wave } \\
\text { number }\end{array}$ & $\begin{array}{l}\text { Wave- } \\
\text { length }\end{array}$ & $\begin{array}{c}\text { Absorp- } \\
\text { tion }\end{array}$ & $\begin{array}{c}\text { Wave } \\
\text { number }\end{array}$ & $\begin{array}{l}\text { Wave- } \\
\text { length }\end{array}$ & $\begin{array}{c}\text { Absorp- } \\
\text { tion }\end{array}$ \\
\hline $\mathrm{cm}^{-1}$ & A & Percent & $\mathrm{cm}^{-1}$ & $A$ & Percent \\
\hline 8656.5 & 11548.9 & 30 & 8567.7 & 11668.5 & 15 \\
\hline 8645.2 & 11563.9 & 30 & 8546.5 & 11697.5 & 15 \\
\hline 8637.6 & 11574.0 & 20 & & & \\
\hline 8628.6 & 11586.2 & 15 & 8523.7 & 11728.8 & 10 \\
\hline \multirow[t]{2}{*}{8616.6} & 11602.3 & 20 & 8509.0 & 11749.0 & 5 \\
\hline & & & 8489.6 & 11775.9 & 5 \\
\hline 8606.5 & 11615.9 & 30 & 8460.9 & 11815.9 & 2 \\
\hline 8601.6 & 11622.6 & 35 & 8421.3 & 11871.4 & 10 \\
\hline 8587.2 & 11642.0 & 25 & & & \\
\hline
\end{tabular}

rotational) symmetry. The centrifugal splitting is roughly independent of the particular vibrational level in question, and is proportional to $J^{2}(J+1)^{2}$. The resonance splitting, proportional to $J(J+1)$, is very dependent on the vibrational state. Thus in the $\nu_{4}$ fundamental [8], the splitting is experimentally observable as low as $J=3$, and has been well accounted for [11] by the secondnamed effect. In the $\nu_{3}$ fundamental [8] however, and equally so in the $2 \nu_{3}$ band here studied, the experimental observation is that the lines of the $P$ and $R$ branches are sharp and narrow, and agree in position and intensity with the predictions of the first-order theory, except for relatively small correction for centrifugal stretching, up to $J=11$. This shows that the second-order interactions leading to splitting are of comparatively minor importance in $2 \nu_{3}$. Presumably such interactions are much more important for the other overtone and combination bands in the region, resulting in bands of much more complex structure which we are unable to interpret.

The following equations represent the observed lines to the required accuracy:

$$
\begin{aligned}
& R \text { branch: } \nu=\nu_{0}+B^{\prime}[(J+1)(J+2)+ \\
& 2 \zeta(J-1)]-B^{\prime \prime} J(J+1)-4 D(J+1)^{3} \\
& \text { Q branch: } \nu=\nu_{0}+B^{\prime}[(J(J+1)-6 \zeta]- \\
& B^{\prime \prime} J(J+1) \\
& P \text { branch: } \nu=\nu_{0}+B^{\prime}[(J-1) J-2 \zeta(J+2)] \\
& B^{\prime \prime} J(J+1)+4 D J^{3} \text {. }
\end{aligned}
$$




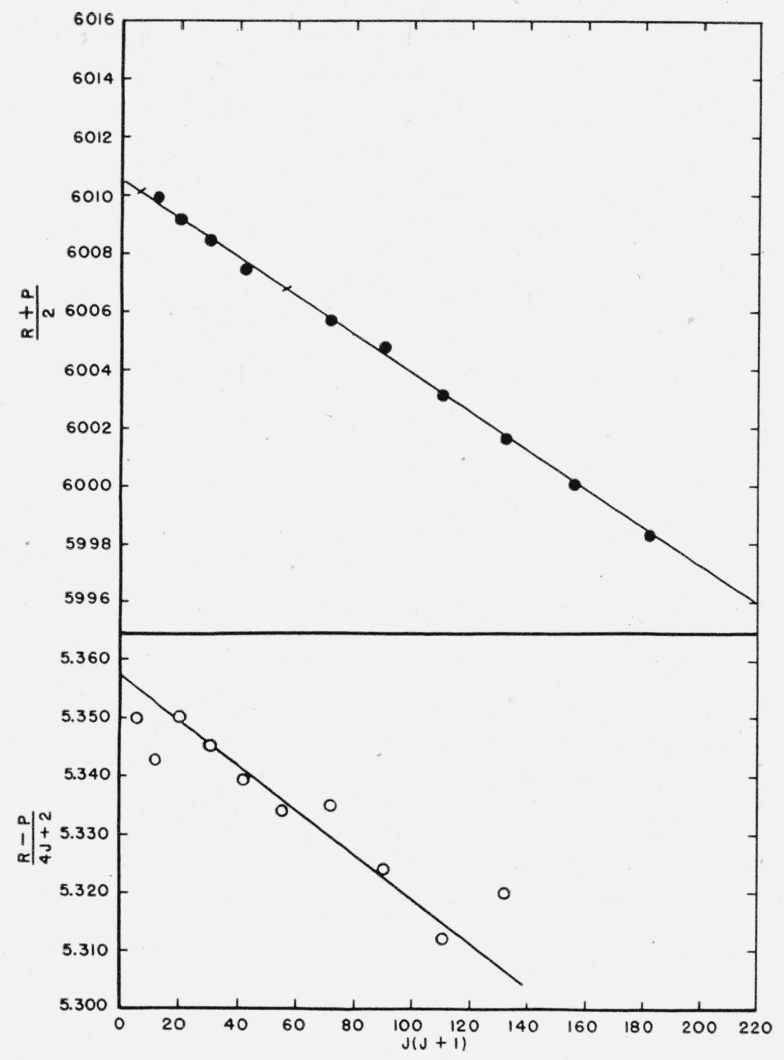

FIgure 5. Plot of the experimental data for the determination of the molecular constants.

Upper plot is based on eq 3, and the lower plot is based on eq 2 .

The following combinations of eq. 1 are useful in deriving the values of the constants:

$$
\begin{array}{r}
\frac{R(J)-P(J)}{4 J+2}=B^{\prime}(1+\zeta)-2 D[J(J+1)+1] \\
\frac{R(J)+P(J)}{2}=Q(J)+B^{\prime}(1+3 \zeta)= \\
\nu_{0}+B^{\prime}(1-3 \zeta)+\left(B^{\prime}-B^{\prime \prime}\right) J(J+1) .
\end{array}
$$

In these equations, $\nu_{0}$ is the band origin, $B^{\prime}$ and $B^{\prime \prime}$ are the effective reciprocal moments of inertia for the upper and ground vibrational levels, respectively, $J$ is the rotational quantum number of the lower level, $\zeta$ is the constant representing the angular momentum due to the upper-state vibration, and $D$ is the constant representing the correction due to centrifugal stretching. As explained above, the effect of the centrifugal forces is both to stretch the molecule, resulting in an equal lowering of all levels of equal $J$, and to distort it, resulting in a splitting of those levels. We have cal- culated both effects for the ground vibrational state, making use of the published formulas [7]; the former effect is larger by a factor of about 5 . Hence it is permissible, for lines where the experimental splitting is unobservable, to make use of a single constant $D$ to correct for the centrifugal stretching, as is the common practice with diatomic or linear molecules.

In Figure 5 are plotted the values of the lefthand sides of eq 2 and 3 as a function of $J(J+1)$. The points fall quite well on straight lines, up to $J=11$, the slopes and intercepts yielding the following combinations of constants: $B^{\prime}(1+\zeta)=$ $5.357 \mathrm{~cm}^{-1}, D=1.9 \times 10^{-4} \mathrm{~cm}^{-1}, \nu_{0}+B^{\prime}(1-3 \xi)=$ $6010.45 \mathrm{~cm}^{-1},\left(B^{\prime}-B^{\prime \prime}\right)=0.066 \mathrm{~cm}^{-1}$.

The value of $D$ is not well determined by the data of figure 5; the slope as drawn is the theoretically calculated value, and also fits the data for the $\nu_{3}$ fundamental.

In order to obtain the values of the individual constants, use is made of data from other bands to obtain $B^{\prime \prime}$, the rotational constant of the ground state, common to all bands. By working up in an analogous way the data of Nielsen and Nielsen [8] on the $\nu_{3}$ fundamental, and of Dickinson, Dillon, and Rasetti [9] on the Raman lines of the same band, we obtain $B^{\prime \prime}=5.244 \mathrm{~cm}^{-1}$. (This compares favorably with the value proposed by Childs [10], $5.252 \mathrm{~cm}^{-1}$, from an analysis of the $\nu_{3}$ and $\nu_{4}$ fundamentals. The perturbations in the latter band, however, make it somewhat less suitable for a precise evaluation). This yields for the $F_{2}$ sub-band of $2 \nu_{3}, B^{\prime}=5.178 \mathrm{~cm}^{-1}, \quad \xi=0.0346$, $\nu_{0}=6005.81 \mathrm{~cm}^{-1}$. The corresponding values for $\nu_{3}$ are $B^{\prime}=5.207 \mathrm{~cm}^{-1}, \quad \zeta=0.0536, \quad \nu_{0}=3018.36$ $\mathrm{cm}^{-1}$. If there were only harmonic terms in the potential energy, the value of $\zeta$ for the two bands should be the same. The observed difference is far beyond the experimental error and must be due to anharmonic terms in the potential energy.

According to eq 3 , there should be a constant difference of $B^{\prime}(1+3 \zeta)=5.72 \mathrm{~cm}^{-1}$ between $[R(J)+P(J)] / 2$ and $Q(J)$. This is very nearly what is observed, although the lines in the $Q$ branch, being not quite fully resolved, are not known with great accuracy. There is, however, a slight but definite anomaly near $J=9$, as shown by the differences in table 3 . It would appear that the $Q$ branch is weakly perturbed by another vibrational level with zero internal angular momentum and a different value of $B^{\prime}$, so that cross- 
TABLE 3. Identified lines in the $2 \nu_{3}$ band of $\mathrm{CH}_{4}$

\begin{tabular}{|c|c|c|c|c|c|c|}
\hline \multirow{2}{*}{$J$} & \multicolumn{2}{|c|}{$R(J)$} & \multicolumn{2}{|c|}{$Q(J)$} & \multicolumn{2}{|c|}{$P(J)$} \\
\hline & $\begin{array}{l}\text { Ob- } \\
\text { served }\end{array}$ & $\begin{array}{l}\text { Calcu- } \\
\text { lated }\end{array}$ & $\begin{array}{l}\text { Ob- } \\
\text { served }\end{array}$ & $\begin{array}{l}\text { Calcu- } \\
\text { lated }\end{array}$ & $\begin{array}{l}\text { Ob- } \\
\text { served }\end{array}$ & $\begin{array}{l}\text { Calcu- } \\
\text { lated }\end{array}$ \\
\hline & $\mathrm{cm}^{-1}$ & $\mathrm{~cm}^{-1}$ & $\mathrm{~cm}^{-1}$ & $\mathrm{~cm}^{-1}$ & $\mathrm{~cm}^{-1}$ & $\mathrm{~cm}^{-1}$ \\
\hline 0 & 6015.8 & 6016.17 & - - . & - n. & -.... & - \\
\hline 1 & 6026.4 & 6026.38 & - . & 6004.60 & 5994.2 & 5994.22 \\
\hline 2 & 6036.8 & 6036.83 & 6004.2 & 6004.34 & 5983.3 & 5983.25 \\
\hline 3 & 6047.0 & 6047.10 & 6003.9 & 6003.94 & 5972.2 & 5972.14 \\
\hline 4 & 6057.3 & 6057.25 & 6003.4 & 6003.41 & 5961.0 & 5960.93 \\
\hline 5 & 6067.2 & 6067. 23 & 6002.7 & 6002.75 & 5949.6 & 5949.58 \\
\hline 6 & 6077.1 & 6077.05 & 6001.9 & 6001.96 & 5938.3 & 5938.13 \\
\hline 7 & 6086.9 & 6086.71 & 6000.8 & 6001.04 & 5926.7 & 5926.59 \\
\hline 8 & 6096.4 & 6096.19 & 5999.6 & 5999. 98 & 5915.0 & 5914.94 \\
\hline 9 & 6106.1 & 6105.52 & 5998.4 & 5998.75 & 5903.4 & 5903.20 \\
\hline 10 & 6114.7 & 6114.66 & 5997.5 & 5997.47 & 5891.6 & 5891.36 \\
\hline 11 & 6123.8 & 6123.61 & 5995.6 & 5996.02 & 5879.5 & 5879.43 \\
\hline 12 & 6133.2 & 6132.39 & 5994.6 & 5994.44 & 5866.9 & 5867.43 \\
\hline 13 & 6142.2 & 6140.94 & 5992.9 & 5992. 72 & 5854.4 & 5855.35 \\
\hline $14^{\circ}$ & 6150.3 & 6149.33 & - n. & - & -.... & ...... \\
\hline 15 & 6158.3 & 6157.52 & - n. & -.... & - & - \\
\hline
\end{tabular}

ing with the $F_{2}{ }^{\circ}$ component occurs near this point. The perturbing level may be the $A_{1}$ sublevel of 2 $\nu_{3}$, or possibly some other combination band with $A_{1}$ symmetry.

In addition to the good agreement between the observed and calculated frequencies, the relative intensities of the lines, especially in the $P$ and $R$ branches, show excellent agreement with those calculated by Jahn [11]. In particular, the relative weakness of the lines with $J^{\prime \prime}=5$ and strength of those with $J^{\prime \prime}=6$ is markedly apparent.

The ultimate goal of a study of the $\mathrm{CH}_{4}$ spectrum is to derive constants that will yield the observed positions, effective moments of inertia, and effective 'S's of all observable overtone and combination bands, and to explain these in terms of a potential function. From the small number of completely interpreted bands this is not yet possible, although the present work is a short step in that direction. Further experimental study under high resolution of $\mathrm{CH}_{4}$ and its deuterated derivatives, and further theoretical studies using a potential function of somewhat greater simplicity than that of Shaffer, Nielsen and Thomas [7], are required. A simplification of the experimental band structure may be expected if measurements are made at low temperatures.

\section{References}

[1] Gerhard Herzberg, Molecular spectra and molecular structure (D. Van Nostrand Co., New York, N. Y., 1945).

[2] Marcel V. Migeotte, Phys. Rev. 73, 519 (1948); R. R. MeMath, O. C. Mohler, and L. Goldberg, Phys. Rev. 73, 1203 (1948).

[3] R. J. Cashman, Proc. Nat. Elec. Conf. 2 (Chicago, 1946).

[4] R. C. Nelson and W. R. Wilson, Proc. Nat. Elec. Conf. 3 (Chicago, 1947).

[5] A. H. Pfund, J. Opt. Soc. Am. \& Rev. Sci. Instr. 14, 337 (1927).

[6] D. M. Dennison and M. Johnston, Phys. Rev. 47, 93 (1935).

[7] W. H. Shaffer, H. H. Nielsen, and L. H. Thomas, Phys. Rev. 56, 895, 1051 (1939).

[8] A. H. Nielsen and H. H. Nielsen, Phys. Rev. 54, 118 (1938).

[9] R. G. Dickinson, R. P. Dillon, and F. Rasetti, Phys. Rev. 34, 582 (1929).

[10] W. H. J. Childs, Proc. Roy. Soc. (London) 153, 555 (1936).

[11] H. A. Jahn, Proc. Roy. Soc. [A], 168, 469, 495 (1938); H. A. Jahn and W. H. J. Childs, Proc. Roy. Soc. [A], 169, 451 (1939).

Washington, October 1, 1948. 